\title{
Modelling and Simulation of Dynamic Connectivity in Different Cislunar Satellite Constellations Networking
}

\author{
Wu Xiaobo ${ }^{1, \text { a }}$, Zhou Xianwei ${ }^{1}$ and Lin Fuhong ${ }^{1}$ \\ ${ }^{1}$ School of Computer and Telecommunication Engineering, University of Science and Technology \\ Beijing, Beijing, China \\ awuxiaobo1980@163.com
}

Keywords: cislunar communication networking; dynamic connectivity; dynamic network modelling; lunar full coverage satellite constellation.

\begin{abstract}
Architecture of cislunar communication networks, which focused on the requirements of lunar full-coverage and continuous cislunar communications, is presented based on Geosynchronous Orbit (GEO) satellite networks relaying. In order to analyze the dynamic connectivity problems, a dynamic network model is constructed in allusion to the time-varying characteristics of cislunar trunk links. Simulations are also performed in this paper with evaluation of dynamic connectivity in different lunar lunar full coverage satellite constellations networking.
\end{abstract}

\section{Introduction}

Demands on lunar satellites networking as well as interconnection with the earth networks are increasing as the development of future lunar exploration activities and the increasing number of lunar satellites and spacecrafts. Space Communication Architecture Working Group (SCAWG) was established by NASA to conduct some fundamental research on the challenges in space communication network [1-4]. Cislunar Communication Networks (CCN) is defined as the expansion interconnection between EN and Lunar Networks (LN) through Cislunar Trunk Links (CTL). The basic requirements of networking are achieving lunar full coverage and ensuring continuous connecting of CTL [2]. Currently, we can categorize 6 types of lunar full coverage satellite constellation: circular polar orbit constellation [5]; inclined circular orbit constellation; walker constellation [6]; equatorial and inclined circular orbit hybrid constellation [7]; elliptic orbit hybrid constellation [8] and Halo orbit constellation [9]. LN constructed by different constellations and orbit parameters will show different dynamic connectivity. Therefore, several critical academic problems, such as modelling the link dynamic of CTL, selecting the necessary component elements to construct CCN architecture and optimizing the connectivity of CTL are raised for further study. These problems are also critical in conducting a further research in network communication protocols. In this paper, the cslunar communication networking is studied. The problem of link dynamic in CTL, which consider the GEO satellites as the relay networks under the distribution restriction of the earth facility, are analyzed.

\section{Dynamic network modelling}

The geographical distribution restriction of the earth facility brings challenges to the continuous cislunar communication. Therefore, we need to draw support from other communication nodes to realize relay forwarding and continuous communication in CCN. In this paper, we choose one earth facility and one GEO relay satellite networks (GRSN), which is composed of three GEO relay satellites, to construct EN. Space communication nodes establish CTLs based on line of sight links, and links cannot be established if there are celestial bodies among communication nodes. There are mainly two kinds of celestial bodies in the CCN, i.e., the earth body and the moon body. With the movement of communication nodes, the pre-establishing CTLs may be interrupted by the celestial bodies. In this case, the other communication nodes will act as the relays for the interrupted nodes to enable a multi-hop relaying and forwarding, as illustrated in Fig. 1. 


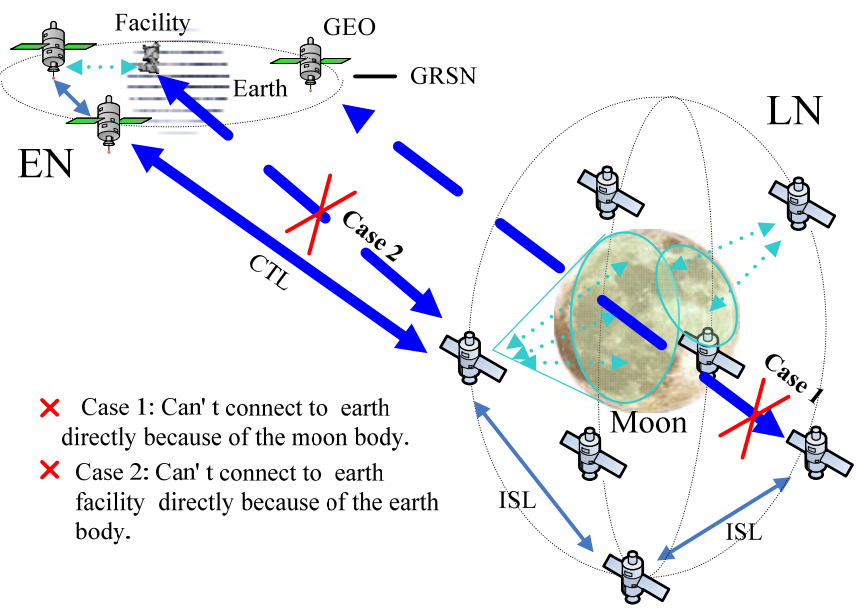

Fig. 1 Architecture of CNN

The topology of CCN can be considered as a dynamic graph $G(t)=(V, E(t))$, in which the communication nodes of the network are expressed as $V=\left\{v_{i} \mid i=1, \ldots, N\right\}$ [10]. The scales of the nodes are unchanged without considering the network extension and the node failure (that means $V$ is not time variant). The set of the dynamic edges $E(t)=\left\{e_{i j}(t) \mid v_{i} \times v_{j} \stackrel{R}{\longrightarrow} e_{i j}(t), v_{i} \in V, v_{j} \in V\right\}$ is used to express the connecting relationship between communication nodes. If the connecting relationship of link is changed with the mobility of network nodes, $E(t)$ becomes time variant.

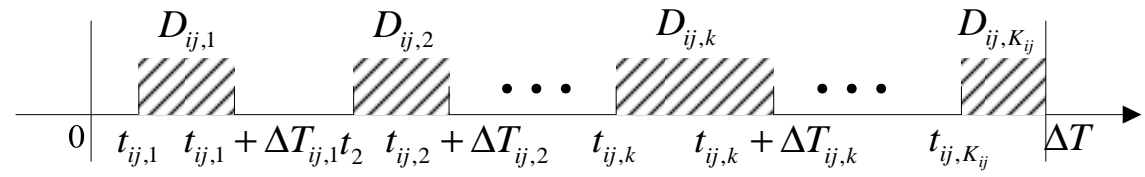

Fig. 2 Time interval of the intermittent connected links

For a given observation time period $T=[0, \Delta T]$, the connectivity state of link $e_{i j}(t)$ is divided into multiple connecting durations (expressed by the shaded areas), and the link can be established at this period, as shown in Fig. 2. The partition of connecting durations should be subject to the minimum duration $d_{\min }$ for communication links establishment. Without loss of generality, let's assume there are $K_{i j}$ connecting durations (as expressed as $D_{i j, 1}, \ldots, D_{i j, K_{i j}}$, where $D_{i j, k}=\left[t_{i j, k}, t_{i j, k}+\Delta T_{i j, k}\right], k=1, \ldots, K_{i j}$ and $\left.\Delta T_{i j, k} \geq d_{\text {min }}\right)$. Time point $t_{i j, 1}, \ldots, t_{i j, K_{i j}}+\Delta T_{K_{i j}}$ can be chosen subject to the constraints of $0 \leq t_{i j, 1}<t_{i j, 1}+\Delta T_{i j, 1}<\cdots<t_{i j, K_{i j}}<t_{i j, K_{i j}}+\Delta T_{i j, K_{i j}} \leq \Delta T$.So the dynamic connectivity of links in the observation time period can be expressed as:

$$
e_{i j}(t)= \begin{cases}1, & t \in \bigcup_{k=1}^{K_{i j}} D_{i j, k} \\ 0, & \text { otherwise }\end{cases}
$$

where 1 and 0 represent the link states of being connected and interrupted, respectively.

\section{Simulation and performance evaluation}

Based on the analysis and modeling aforementioned, the simulation of CCN is performed by using software tools of STK 8.1.1. EN and LN are also established in this simulation scenario, where EN is composed of an earth facility and three uniformly distributed GEO satellites. The earth facility is under the coverage of one GEO satellite, and the minimum elevation is set to be $10^{\circ}$. LN selects six kinds of constellations individually, as shown in table 1 (a)-(f). The minimum duration $d_{\text {min }}$ is set to 600 seconds.

Table 1 Parameters of lunar satellite constellations 


\begin{tabular}{|c|c|c|c|c|c|c|c|c|}
\hline Label & Constellations & Satellites & Planes & $\begin{array}{l}\text { Semi-major } \\
\text { Axis }(\mathrm{km})\end{array}$ & Eccentricity & $\begin{array}{c}\text { Inclination } \\
\text { (degree) }\end{array}$ & $\begin{array}{l}\text { Argument } \\
\text { of Perigee }\end{array}$ & $\begin{array}{c}\text { RAAN } \\
\text { (degree) }\end{array}$ \\
\hline \multirow{2}{*}{ (a) } & \multirow{2}{*}{ Polar orbits } & \multirow{2}{*}{6} & \multirow{2}{*}{2} & \multirow{2}{*}{9250} & \multirow{2}{*}{0} & \multirow{2}{*}{$90^{\circ}$} & \multirow{2}{*}{$0^{\circ}$} & $0^{\circ}$ \\
\hline & & & & & & & & $90^{\circ}$ \\
\hline \multirow{2}{*}{ (b) } & \multirow{2}{*}{ Inclined orbits } & \multirow{2}{*}{6} & \multirow{2}{*}{2} & \multirow{2}{*}{8050} & \multirow{2}{*}{0} & $52.2^{\circ}$ & \multirow{2}{*}{$0^{\circ}$} & \multirow{2}{*}{$0^{\circ}$} \\
\hline & & & & & & $142.2^{\circ}$ & & \\
\hline (c) & Walker & 5 & 5 & 9150 & 0 & $43.7^{\circ}$ & $360^{\circ} / 5$ & $360^{\circ} / 5$ \\
\hline (d) & Walker & 6 & 2 & 8050 & 0 & $52.2^{\circ}$ & $360 \% / 6$ & $360^{\circ} / 6$ \\
\hline \multirow{2}{*}{ (e) } & \multirow{2}{*}{$\begin{array}{l}\text { Inclined orbits \& } \\
\text { Equatorial orbits }\end{array}$} & \multirow{2}{*}{6} & \multirow{2}{*}{5} & \multirow{2}{*}{8050} & \multirow{2}{*}{0} & $52.2^{\circ}$ & $360^{\circ} / 4$ & $360^{\circ} / 4$ \\
\hline & & & & & & $0^{\circ}$ & $0^{\circ}$ & $0^{\circ}$ \\
\hline \multirow{3}{*}{ (f) } & \multirow{3}{*}{$\begin{array}{l}\text { Elliptical orbits \& } \\
\text { Inclined orbits }\end{array}$} & \multirow{3}{*}{7} & \multirow{3}{*}{3} & \multirow{2}{*}{6541} & \multirow{2}{*}{0.6} & \multirow{2}{*}{$56.1^{\circ}$} & $90^{\circ}$ & \multirow{2}{*}{$0^{\circ}$} \\
\hline & & & & & & & $270^{\circ}$ & \\
\hline & & & & 11575 & 0 & $33.9^{\circ}$ & $0^{\circ}$ & $180^{\circ}$ \\
\hline
\end{tabular}

In this paper, we choose connectivity rate and interrupt frequency, as indicators to estimate the stability of the dynamic CTLs. The connectivity rate of links is the indicator of measuring the connecting duration of communication link among nodes, and this parameter is reference to describe the connectivity stability of dynamic links. It can mainly reflect the time-domain occupation ratio of the link connectivity state, and is expressed as:

$$
R\left(e_{i j}(t), \Delta T\right)=\sum_{k=1}^{K_{i j}} \Delta T_{i j, k} / \Delta T, e_{i j}(t) \in E_{V_{E}, V_{M}}(t)
$$

Since dynamic links have characteristics of intermittent connectivity, for each communication traffic stream, it requires each interrupted link to find a new visible node and switch traffic to the new link. Therefore, the link stability can be reflected by the number of interruption. In this paper, we replace the number of interruption with the number of connecting durations $K$ in observation time period to reflect the number of connection established. The interrupt frequency is represented as:

$$
I\left(e_{i j}(t), \Delta T\right)=K_{i j} / \Delta T, e_{i j}(t) \in E_{V_{E}, V_{M}}(t)
$$

The numerical results of dynamic CTL in six kinds of LN are shown in Fig. 3 and Fig. 4.

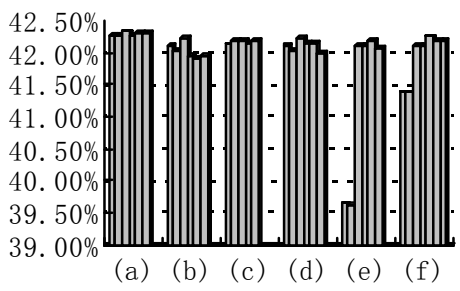

(a) To Facility

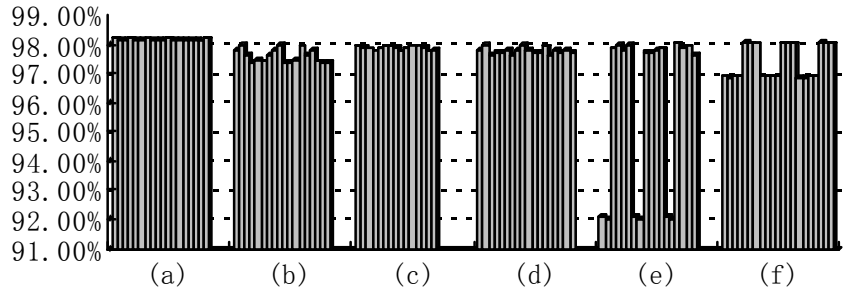

(b) To GRSN

Fig. 3 Connectivity rate of dynamic CTL

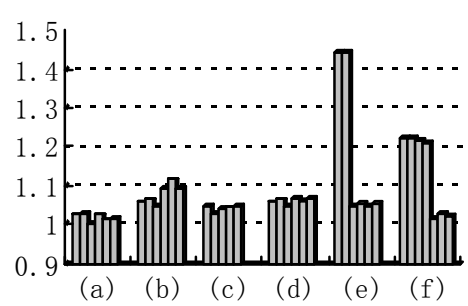

(a) To Facility

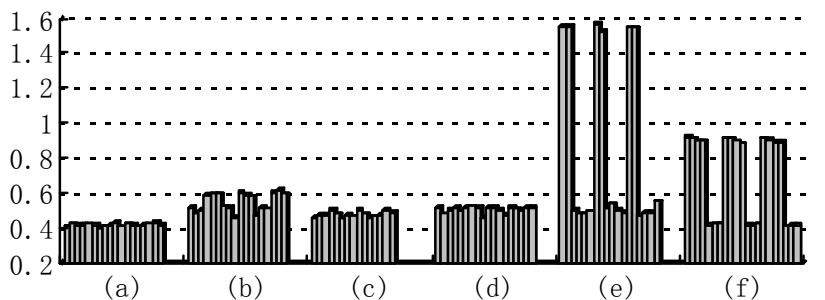

(b) To GRSN

Fig. 4 Interruption frequency of dynamic CTL

From the simulation results we know that above $80 \%$ of the connectivity rate of the six candidate constellations can be obtained, and the interrupt frequency is less than one time per earth day. For the four kinds of constellations comprise six lunar satellites, the orbit altitude of constellation (a) is larger than others and the optimum stability of CTL can be obtained. Constellation (d) is composed of uniformly distributed satellites and its stability is in the third place among all these constellations. Constellation (b) has the same space structure as that of constellation (a), but the difference of inclination makes its CTLs stability are in the fourth place. Constellation (e) selects two lunar equator satellites to comprise the hybrid constellation so that the blocking from the lunar body is serious, and 
the worst CTL stability is then obtained. Hybrid constellation (f) is composed of elliptic orbits and inclined orbits with seven lunar satellites. The elliptical orbit is frozen orbit, which has a higher satellite stability and better coverage of the lunar polar region. However, it can be seen from the results that the elliptic orbit satellites have poor CTL stability due to the non-uniform motion of satellites, and its stability of CTL is only better than that of constellation (e). It is worth noting that the walker constellation (c) realizes lunar full coverage with minimum five satellites, and the CTL stability is only inferior to that of constellation (a), but its capability of coverage of polar region is worse than that of others. On the other hand, walker constellation (c) is composed of five orbital planes, the additional expenditure should be considered in the process of satellites injection. In summary, constellations (a), (c), and (d) have remarkable advantage on CTL stability, as proven by the comparison and analysis aforementioned, and therefore, it is the ideal choice of constellation for LN. Besides, the circular polar orbit constellation has stronger capability for lunar polar coverage, while the walker constellation can achieve complete intra-network connectivity. In terms of the practical process of architecture engineering, there should be a trade-off between various factors (such as the connectivity of both inter-network and intra-network, expenditure of satellites, capability of coverage, and invulnerability, etc) according to scientific missions for lunar exploration.

\section{Conclusions}

In order to achieve lunar full coverage and continuous cislunar communications under the geographical restrictions of constructing earth facilities, a networking scheme base on GRSN relaying was proposed in this paper. The dynamic connectivity problem between LN and EN was mathematically formulated through modelling of the dynamic links. In simulation experiments, we evaluated the stability of cislunar trunk links in six different typical constellations by two evaluation indicators. The numerical results show that circular polar orbit constellation and walker constellations have remarkable advantage on CTL stability over the other schemes, and therefore, they can be chosen as the ideal candidates of constellation for lunar Satellite Constellations Networking. Through analyzing of the dynamic network connectivity, all results in this paper provide theoretic reference and basic data to research on the network architecture of cislunar communication and multi-hop routing and forwarding mechanism in the future works.

\section{Acknowledgment}

The research was supported by the National Nature Science Foundation Project of P. R. China (61170014), the National Research Foundation for the Doctoral Program of Higher Education of China under Grant No.20090006110014, the Foundation for Key Program of Ministry of Education, China (Grant No. 311007).

\section{References}

[1] J. Schier, J. Rush, W. Williams, et al. Space Communication Architecture Supporting Exploration and Science: Plans and Studies for 2010-2030 [C]. AIAA 1st Space Exploration Conference: Jan 30-Feb 1, 2005, Orlando, USA.

[2] Space Communication Architecture Working Group: NASA Space Communication and Navigation Architecture Recommendations for 2005-2030 [R]. Final Report, May 15, 2006.

[3] K. Bhasin, A. Hackenberg, R. Slywczak, et al. Lunar Relay Satellite Network for Space Exploration: Architecture, Technologies and Challenges [C]. Proceedings 24nd AIAA International Communications Satellite Systems Conference: Jun 12-14, 2006, San Diego, California, USA.

[4] K. Bhasin, C. Putt, J. Hayden, et al. Architecting the Communication and Navigation Networks for NASA's Space Exploration Systems [C]. System of Systems Engineering, 2007. SoSE '07. IEEE International Conference on: Apr 16-18, 2007, San Antonio, Texas, USA. 2007: 1-6. 
[5] L. Rider. Optimized polar orbit constellations for redundant earth coverage [J]. Journal of the Astronautical Sciences, 1985, 33: 147-161.

[6] J. Walker. Some circular orbit patterns providing continuous whole earth coverage [J]. Journal of the British Interplanetary Society, 1971, (24): 369-384.

[7] T. Lang, J. Meyer. A New Six Satellite Constellation for Optimal Continuous Global Coverage [C]. AAS/AIAA Space Flight Mechanics Conference: Feb 13-15, 1995, Albuquerque, New Mexico, USA.

[8] T. Ely, E. Lieb. Constellations of Elliptical Inclined Lunar Orbits Providing Polar and Global Coverage [C]. AAS/AIAA Spaceflight Mechanics Meeting: Aug 7-11, 2005, South Lake Tahoe, California, USA.

[9] K. Hamera, T.Mosher, M. Gefreh, et al. An Evolvable Lunar Communication and Navigation Constellation Concept [C]. Proceedings of the IEEE Aerospace Engineering Conference: Mar 1-8, 2008, Big Sky, Montana, Canada, 2008: 1-20.

[10] E. Prisner, Graph Dynamics [M]. Harlow, U.K. Longman House, 1995. 